\title{
Identification of indels in next-generation sequencing data
}

\author{
Aakrosh Ratan ${ }^{1,3^{*}}$, Thomas L Olson ${ }^{2}$, Thomas P Loughran $\mathrm{Jr}^{2}$ and Webb Miller ${ }^{1}$
}

\begin{abstract}
Background: The discovery and mapping of genomic variants is an essential step in most analysis done using sequencing reads. There are a number of mature software packages and associated pipelines that can identify single nucleotide polymorphisms (SNPS) with a high degree of concordance. However, the same cannot be said for tools that are used to identify the other types of variants. Indels represent the second most frequent class of variants in the human genome, after single nucleotide polymorphisms. The reliable detection of indels is still a challenging problem, especially for variants that are longer than a few bases.

Results: We have developed a set of algorithms and heuristics collectively called indelMINER to identify indels from whole genome resequencing datasets using paired-end reads. indelMINER uses a split-read approach to identify the precise breakpoints for indels of size less than a user specified threshold, and supplements that with a paired-end approach to identify larger variants that are frequently missed with the split-read approach. We use simulated and real datasets to show that an implementation of the algorithm performs favorably when compared to several existing tools.

Conclusions: indeIMINER can be used effectively to identify indels in whole-genome resequencing projects. The output is provided in the VCF format along with additional information about the variant, including information about its presence or absence in another sample. The source code and documentation for indeIMINER can be freely downloaded from www.bx.psu.edu/miller_lab/indelMINER.tar.gz.
\end{abstract}

Keywords: Indels, Variants, Sequencing analysis

\section{Background}

Genetic differences between individuals are encoded as local changes consisting of substitutions and small indels that alter a few base pairs, and large-scale changes that consist of larger indels, rearrangements and copy number variations. Whole genome sequencing using NGS technologies offers a unique opportunity to study these variations and enable a better understanding of genome function and diversity. There are a number of mature software packages and associated pipelines that can identify single nucleotide polymorphisms (SNPs) with a high degree of concordance [1]. However, the same cannot be

\footnotetext{
* Correspondence: ratan@bx.psu.edu

${ }^{1}$ Center for Comparative Genomics and Bioinformatics, Pennsylvania State

University, 506, Wartik Laboratory, University Park, PA 16802, USA

${ }^{3}$ Department of Public Health Sciences and Center for Public Health

Genomics, University of Virginia, Charlottesville, VA 22908, USA

Full list of author information is available at the end of the article
}

said for tools that are used to identify the other sources of variation.

Indels are the most common structural variant that contribute to pathogenesis of disease [2], gene expression and functionality. Current approaches to identify indels include de-novo assembly of unaligned reads [3], read splitting [4,5], depth of coverage analysis [6] and analysis of insert size inconsistencies. Each of these approaches has their own strengths and weaknesses. For example, even though de-novo assembly offers the best opportunity to accurately call these variants, assembly with short reads is a challenging problem that requires significant computational resources. Similarly split-read approaches perform with a high degree of accuracy for short and medium sized indels, but the false-negative rate increases significantly with increase in size of the variations. Paired-end read and depth of coverage approaches frequently miss small indels, and are unable to predict the breakpoints accurately. We believe a hybrid 
strategy that integrates the information using more than one of the above approaches is required to identify these indels with a high degree of sensitivity and specificity.

Here we present indelMINER, a method that uses a combination of split-read and paired-end approaches to identify the breakpoints of insertions and deletions. The identified indels can be annotated with additional information such as the depth of coverage across the predicted breakpoints, and the list can be subsequently filtered to generate a high quality subset of variants. In addition to identification of indels, indelMINER can also be used to investigate the absence or presence of support for a set of indels in another sample. This is valuable in investigation of normal/tumor pairs as well in cases where several individuals of a family are sequenced to identify de-novo changes in the proband, and a novel feature of indelMINER. We present the results of using indelMINER on simulated data as well as real data from the individual NA18507 and a cancer genome dataset. We compare the performance and results of indelMINER to previously published results from several other similar tools.

\section{Results}

\section{Simulated dataset}

In order to calculate the sensitivity and specificity of our method, and compare it to that of a few other popular tools, we implanted 3,723 known homozygous deletions, and 3,777 known homozygous insertions [7] into chromosome 22 of the human genome. $100 \mathrm{bp}$ long paired-end (average insert distance 500 bps, s.d. 30 bps) Illumina reads were simulated from this modified sequence using pIRS [8], such that each nucleotide on the reference was covered 20 times on an average. The reads were mapped to the human reference chromosome 22 using BWA [9] version 0.5.9, with the default parameters. The resulting BAM file was sorted based on the chromosomal coordinates, and the reads were realigned around putative indels using IndelRealigner tool from the GATK suite [10].

We ran SAMtools [11], PINDEL [4], PRISM [5] and indelMINER on this dataset and the results are summarized in Table 1. The indels identified by the tools were compared to the true set, and in case of deletions a call was marked as validated if there was a reciprocal overlap with at least half of the actual deletion. The details of

\begin{tabular}{|c|c|c|c|}
\hline SV caller & Observed indels & False-positives & False-negatives \\
\hline SAMtools & 6,491 & $172(2.65 \%)$ & 1181 \\
\hline PINDEL & 7,239 & $328(4.53 \%)$ & 589 \\
\hline PRISM & 7,406 & $301(4.06 \%)$ & 395 \\
\hline indelMINER & 7,365 & 263 (3.57\%) & 398 \\
\hline
\end{tabular}

the arguments and parameters used for this experiment are detailed in the Additional file 1. SAMtools exhibits the lowest false-positive rate for this dataset (2.65\%), but its false-negative rate is significantly higher when compared to the other software. Out of the remaining tools, indelMINER exhibits the lowest false-positive rate $3.57 \%$ compared to $4.06 \%$ for PRISM and $4.53 \%$ for PINDEL) as well a false-negative rate that is significantly lower than PINDEL (10.54\% for indelMINER, 15.59\% for PINDEL), and comparable to that of PRISM (10.46\%).

\section{Real dataset}

We used about 28-fold data corresponding to the Yoruban HapMap individual NA18507 (Accession: SRX016231) to evaluate indelMINER on real data. The same sample has been characterized in multiple studies $[4,5,12,13]$ and sequenced using multiple platforms [14,15], making it an ideal test case to compare the results of indelMINER. We downloaded the fastq reads for the HapMap individual from the Short read archive (Accession: SRX016231). These $101 \mathrm{bp}$ reads were generated using the standard Illumina paired-end library protocol, with an average insert length of about $500 \mathrm{bps}$. We aligned these reads to the hg19 reference sequence using BWA version 0.5.9 with the default parameters except -q 15, which was used to trim the low quality segment of the read down to 35 bps at the 3' end. The reads around putative indels were realigned using GATK IndelRealigner, followed by use of MarkDuplicates (http://picard.sourceforge.net) to flag the potential PCR duplicates. The resulting BAM file was used to identify indels using SAMtools, PINDEL, PRISM and indelMINER (See Additional file 1).

For the NA18507 genome, indelMINER detected 643,636 indels (347,590 deletions and 296,046 insertions). Additional file 1: Figure S1 shows the length distribution of the identified indels and Additional file 1: Figure S2 shows their distribution across the human chromosomes, which correlates well to the amount of DNA present in the chromosomes. 313 of the identified indels overlap with the protein coding exons corresponding to the set of RefSeq [16] genes. $44.81 \%$ of these coding indels are of lengths that are a multiple of 3 . This is in close concordance with previous studies $[17,18]$ that have reported that in-frame indels should constitute about $50 \%-60 \%$ of all coding indels. $412,001(64.01 \%)$ of these indels identified using indelMINER were also found in dbSNP version 137 and $454,120(70.55 \%)$ of them were found in the Database of Genomic Variants (DGV). 220,434 (34.25\%) of the variants were also identified in the Phase 1 release 3 of the 1000 genomes project in African samples.

We also compared the variants identified using indelMINER to those identified using SAMtools, PINDEL and PRISM. Figure 1 shows a comparison of the variants called by the various tools using the same read alignments. Two calls 


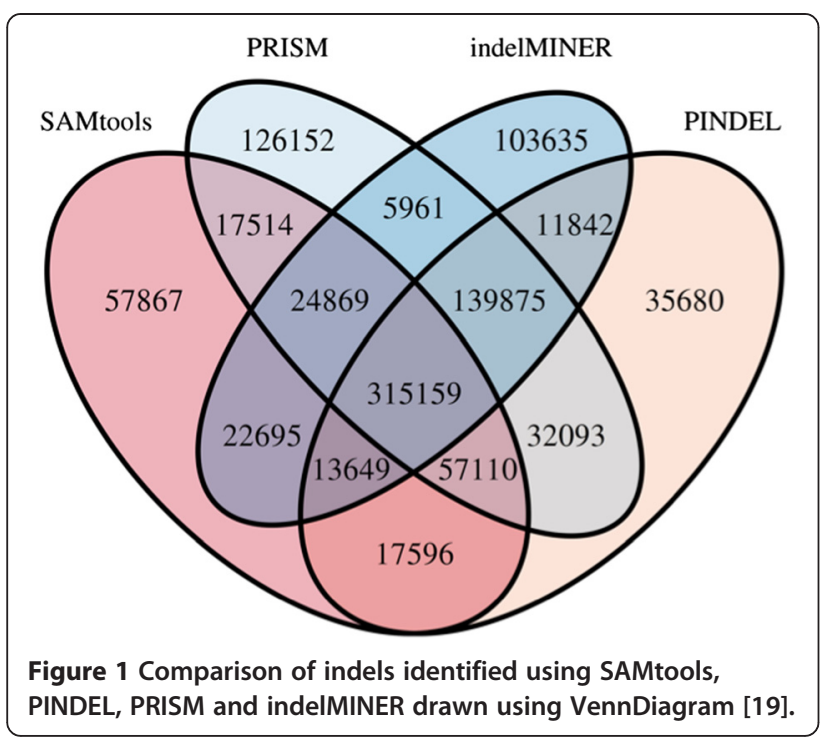

were marked as an overlap if they had a reciprocal overlap greater than $50 \%$ of the breakpoint range. All of the included software agreed on 315,159 of the indels, whereas about 658,363 of the indels were supported by at least two of the software we looked at as part of this study.

\section{Cancer normal/tumor pair}

All cancers arise as a result of accumulation of mutations that confer growth advantage. The advent of next-generation sequencing provides a powerful and cost-effective tool to characterize these genome-wide changes. The primary tumor tissue and adjacent or distal normal tissue are frequently sequenced and analyzed to identify germline and rare somatic mutations. The first step in such an analysis is to identify the mutations that are unique to the cancer. Issues such as normal DNA contamination of tumor DNA complicate the analysis by reducing the tumor variant allele frequency.

Large granular lymphocyte (LGL) leukemia is characterized by a clonal expansion of either $\mathrm{CD}^{+}$cytotoxic $\mathrm{T}$ or $\mathrm{CD}^{-} \mathrm{NK}$ cells, and is frequently associated with autoimmune diseases such as rheumatoid arthritis [20,21]. A patient was consented under Institutional Review Board protocols initiated at the Pennsylvania State University and continuing at the University of Virginia in accordance with the Declaration of Helsinki. The patient consented to inclusion in an LGL Leukemia patient registry which permits the publication of de-identified patient characteristics and an additional addendum consenting to next generation sequencing and the public deposition of data derived therefrom. We sequenced the peripheral blood and matched saliva from a patient diagnosed with LGL, to a coverage of 29-fold and 17-fold respectively (Additional file 1: Figure S3). We used indelMINER to (a) identify indels in the blood sample and (b) investigate and tag those indels based on their presence or absence in the matched saliva sample. indelMINER identified 575,426 indels in the blood sample, out of which 572,188 of them were also observed in the saliva. Indelocator (https://www. broadinstitute.org/cancer/cga/indelocator) has been used in earlier studies [22] to identify indels in normal/tumor pairs. We used Indelocator on the same dataset, and it identified 478,534 indels in the blood sample, 438,331 of which were also observed in the matching normal sample. We found that $392,512(82.02 \%)$ of the indels found by Indelocator were also found by indelMINER, whereas the remaining indels were observed by only one of the two software tools. We randomly selected 10 indels that were identified by indelMINER but not identified by Indelocator for validation (Additional file 2). We were not able to design a reliable pair of primers for 5 of the indels due to their location in low-complexity regions or repeat regions in the human genome. 4 of the remaining 5 indels were validated using Sanger sequencing, including a large deletion spanning 350 bases (Figure 2).

\section{Discussion and conclusions}

Recent studies have reported on the concordance of singlenucleotide variants identified using different software tools [1] as well using different sequencing platforms [23]. The fraction of polymorphic sites where all platforms/tools agree varies between $70-90 \%$ for the SNP calls. Often the overlap of predicted indels between different methods is much lower, indicating that none of the methods offer a comprehensive satisfactory solution.

indelMINER uses a combination of approaches to identify indels of arbitrary size from paired-end short reads. It can predict the exact breakpoint for small and medium size indels, and the approximate breakpoints for the larger deletions. The performance of the algorithm degrades in regions where a single short-read covers multiple indels, as well as in regions where the mapping quality of the sequences is low. A de novo assembly approach has been shown to be more suitable in a large fraction of such regions. The current version of indelMINER can only handle indels; however the same algorithm can be extended to handle other types of structural variants, in a manner similar to PINDEL and PRISM. We do not use sequences where both reads from the same fragment align with a mapping quality of zero, i.e., cases where neither of the mates can be aligned unambiguously in finding the indels. If one of the reads can be aligned unambiguously, then indelMINER can use that information to split and align the second read. As explained earlier, we do use such sequences that align ambiguously in the mode where we are just looking to tag the presence or absence of a variant. When tagging the presence or absence of indels in sample $\mathrm{B}$ indelMINER uses all the alignments including the 


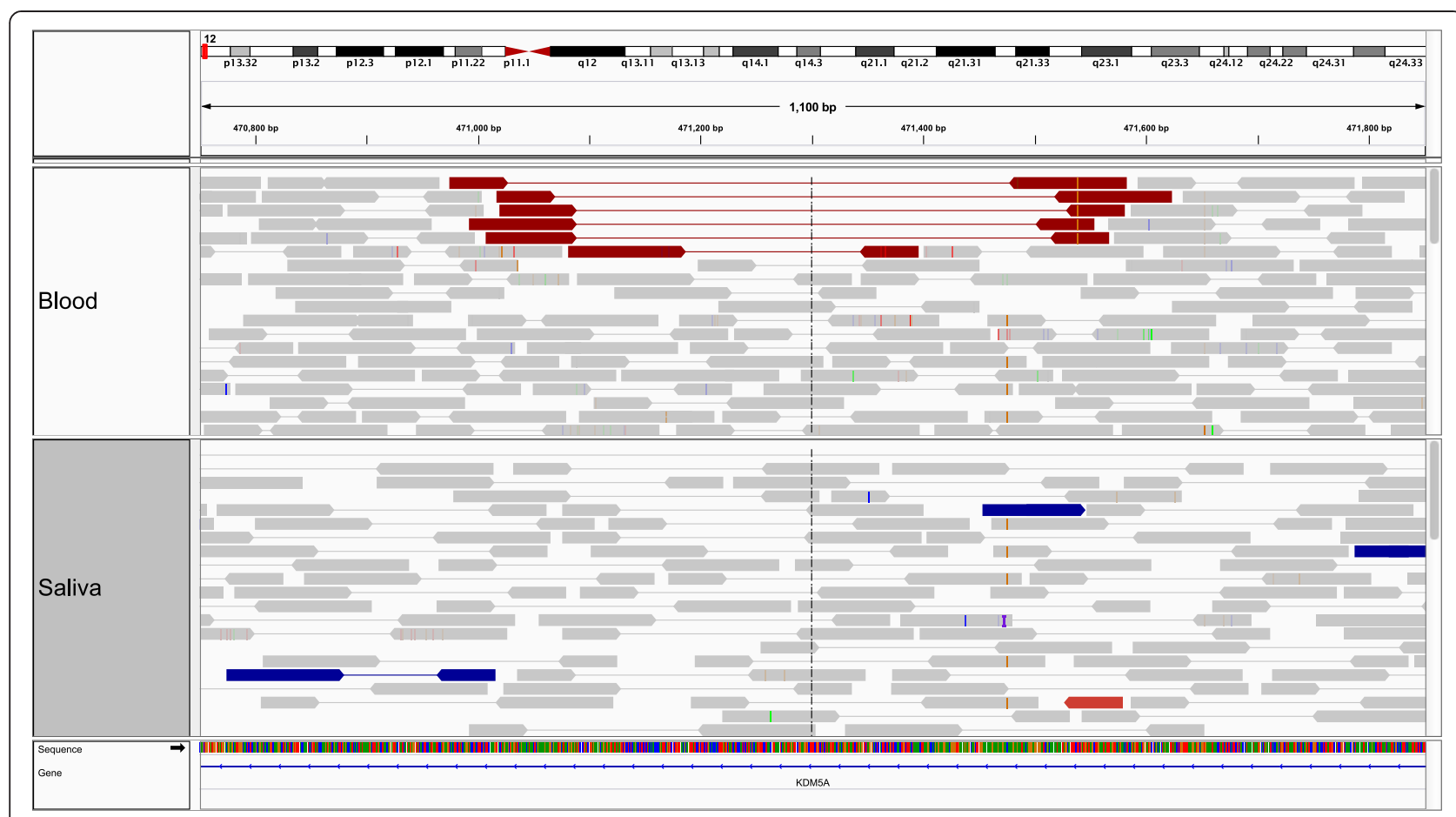

Figure 2 IGV snapshot of the alignments showing the deletion chr12:471,091-471,494 in the blood sample (top half of the plot) and the absence of the deletion in the saliva sample (bottom half of the plot).

secondary alignments to check against the indels found in sample A.

We used both simulated and real data to show that indelMINER has low false-positives and a low false-negative rate when compared to several other tools in the same category. indelMINER can also be used in study of normal/ tumor pairs, and in studies where multiple individuals from the same family are being sequenced. The PCR validations confirm the accuracy and sensitivity of indelMINER, and its ability to identify indels in high-throughput sequencing datasets.

\section{Methods}

\section{Overview}

indelMINER relies on a combination of split-read and paired-end read approaches to identify indels from a BAM file for a sample (Figure 3). Even though it can be run on any coordinate sorted BAM file, we recommend running the GATK IndelRealigner [10] on it prior to running indelMINER. This local realignment serves to transform regions with misalignments due to indels into clean reads containing a consensus indel that can be then easily identified. The cleaned reads are analyzed in order of their alignments to the reference sequence, and segments of candidate reads are realigned within a specified diagonal band [24], identified using a fast $k$-mer comparison of the read and the reference sequence. These alignments are collected and used to identify candidate insertions and deletions. The identified variants are annotated with additional information pertaining to the region within the breakpoints, including the average depth of coverage, the RMS mapping quality of reads, and the count of reads with a mapping quality equal to zero. These can be used to filter the calls to obtain a more reliable set of differences between the target and the reference genome. Here we describe each of the steps in greater detail.

\section{Definitions}

First we define a few terms that will be used in the description of the workflow and algorithms used in indelMINER.

1. A read group $R(a, b, o)$ is defined as a set of paired-end sequences that are the product of a single lane or barcode of a sequencing run. The expected outer distance for the pairs in this group is described by the interval $[a, b]$ and the expected relative orientation is given by $o$, where $o \in\left[{ }^{\prime}++,{ }^{\prime}+\right.$ ' $\left.^{\prime},{ }^{-}-+,{ }^{\prime},-'\right]$. The first symbol represents the orientation of the mate that comes earlier on the chromosomal co-ordinates. For example, the expected relative orientation for Illumina paired-end reads is ' +- '.

2. A paired-end sequence $P(r 1, r 2, r, o, i)$ consists of two reads $r 1$ and $r 2$ that are sequenced from the same DNA fragment. The paired-end fragment belongs to the read group $r$, and $o$ and $i$ refer to the 
Identification of candidate reads

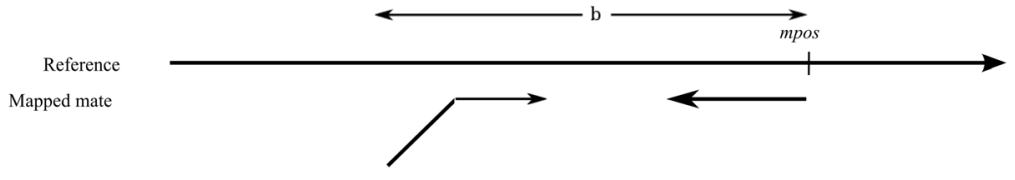

b)
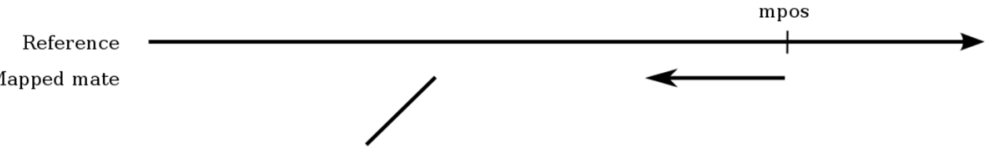

c)

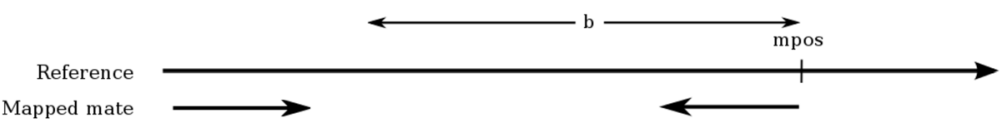

Identification of diagonal

$$
\text { Reference }
$$

Mapped mate

Unmapped mate
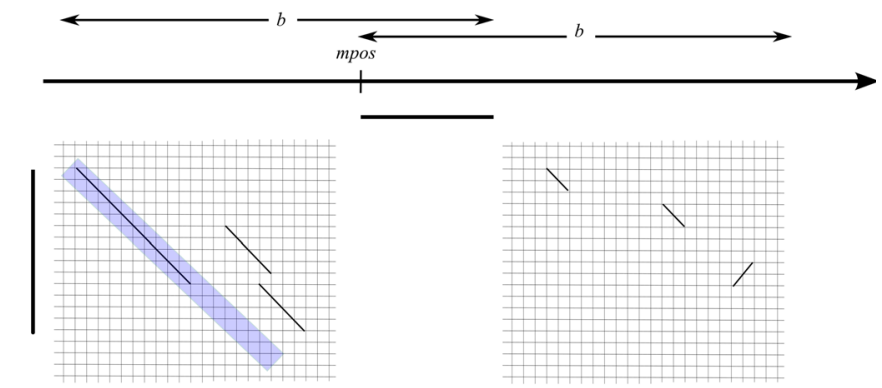

Split read alignment

$$
\begin{aligned}
& \text { Reference } \\
& \text { Unmapped mate }
\end{aligned}
$$

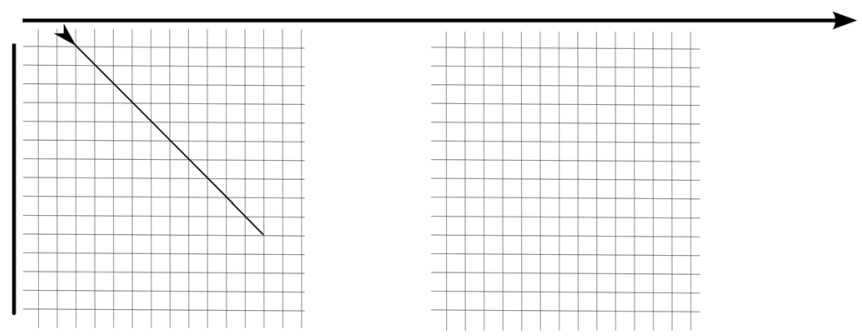

Identification of indel

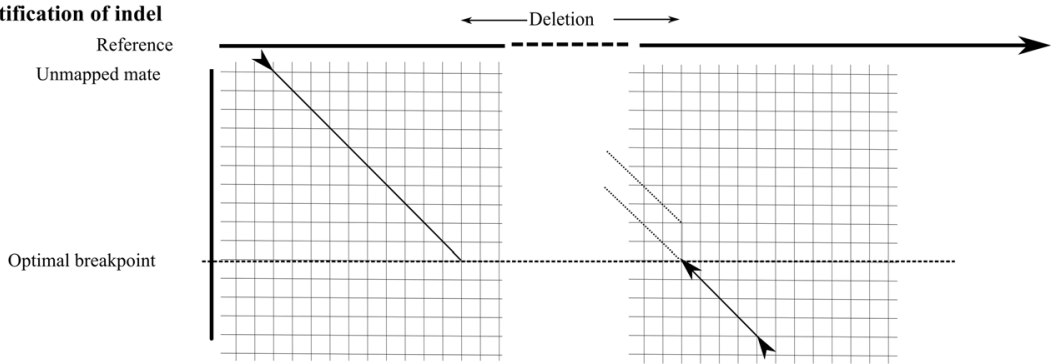

Figure 3 (See legend on next page.) 
(See figure on previous page.)

Figure 3 Overview of the indeIMINER algorithm. Panel titled "Identification of candidate reads" shows three of the cases when a read is identified for realignment or paired-end analysis. (a) shows a case when mates align with the expected orientation but one of the mates is only partially aligned, (b) shows a case when one mate from a fragment aligns to a location mpos on the reference, while the other mate does not align, and (c) shows the case when both mates align with the expected relative orientation, but the outer distance constraint is violated. Panel titled "Identification of diagonal" shows the various alignments of the unaligned mate using k-mer comparisons, and the subsequent selection of one of the diagonals based on alignment score and distance from mpos. Panel "Split read alignment" shows the extension of the chosen diagonal, and the panel "Identification of indel" shows the alignment and extension of the remaining sequence from the unaligned mate, to a region around mpos selected based on a user-specified threshold.

relative orientation and the outer distance of $r 1$ and $r 2$ when both of them are aligned to a reference sequence. The first symbol in $o$ defines the orientation of $r 1$, and the second symbol defines the orientation of $r$.

3. maxsrdelsize and maxpedelsize are user specified thresholds that refer to the maximum size of the deletion that we want to identify using split read and paired-end read approaches respectively.

\section{Identification of candidate reads}

The reads in the BAM file are analyzed in order of their alignment to the reference sequence. A read $r 1(r 2)$ of a pair $P(r 1, r 2, r, o, i)$ is selected for split read alignment if any of the following conditions is satisfied

a) $P(r 1, r 2, r, o, i)$ is properly paired $\left(o \in\left[{ }^{\prime}+-'\right]\right.$ for Illumina PE reads, and $a \leq i \leq b$ where $r=R(a, b, o)$ ), and $r 1$ (r2) aligns to the reference with one or more indels, or has a unaligned/soft-clipped segment in it. In other words, these are the reads that align to the reference genome with the expected orientation and outer distance, but one of the mates is either aligned partially, or aligned to the reference genome with one or more gaps (Figure 3, Identification of candidate reads (a)).

b) The mate $r 2(r 1)$ is aligned but $r 1(r 2)$ is unaligned (Figure 3, Identification of candidate reads (b)).

We also collect the pairs $P(r 1, r 2, r, o, i)$ where $r 1$ and $r 2$ align to the reference with the expected orientation, but the insert length constraints are not satisfied i.e. $(i<a)$ or $(i>b)$, where $r=R(a, b, o)$ for a separate pairedend read analysis (Figure 3, Identification of candidate reads $(c)$ ).

\section{Split read alignment of reads}

As discussed above, a read $r 1$ that is selected for splitread analysis has a mate read $r 2$ that aligns to the reference sequence at a position denoted by mpos (Figure 3, Identification of candidate reads). The read $r 1$ is now aligned to the reference within the interval [mpos $-b$, mpos $+b]$, where $b$ refers to the maximum expected outer distance for their read group (Figure 3, Identification of diagonal, Split read alignment). If one end of the read aligns to position pos in the above interval, then we attempt to align the read from the other end within the interval [pos, pos + maxsrdelsize] or [pos - maxsrdelsize, pos], depending on the relative orientation of $r 1$ and $r 2$ (Figure 3, Identification of indel). If that fails then we check to see if the unaligned segment of $r 1$ is a candidate insertion. The above alignments proceed in two steps. First, we use a $k$-mer comparison of the read sequence to the candidate reference segment to find the best diagonal band i.e. the diagonal band where the read and the reference share the most number of unique $k$-mers. The alignments are then performed using a strategy that requires only $O(N W)$ computation time and $O(N)$ space, where $\mathrm{N}$ is the length of the shorter of the two subsequences and $W$ is the width of the band [24]. Each split read where both read ends can be aligned in a way that they support an indel, are saved for further analysis.

\section{Identification of indels}

In this step, we collect all the candidate variants $V(e 1, e 2)$, where $e 1$ and $e 2$ refer to either the two split halves as a result of the realignment in the previous step, or refer to the two reads from the same fragment that did not satisfy the outer distance constraints. We create a graph $\mathrm{G}$ and represent every candidate variant supported by a split or paired-end read, as a vertex. Two vertices are joined by an edge if they support the same variant in the target genome. If the two vertices represent split-reads, then the only condition for an edge between them is that they support the same breakpoints. If the two vertices represent mates that do not satisfy distance constraints, then an edge can be drawn between them if the resulting breakpoints from the two variants do not violate the outer distance constraint for reads represented by $V 1$ and $V 2$.

Each clique in the graph should now represent a variant in the target genome. However due to errors in sequencing, ambiguous alignments, ploidy, incompleteness and inaccuracies in the reference genome, a significant fraction of these subgraphs are not fully connected. So instead of restricting the definition of a variant to a clique, we consider each connected component in the graph $\mathrm{G}$ to represent a variant in the target genome, and the vertices in the connected component to represent the evidence that 
supports that particular variant. We heuristically identify a maximal clique in each connected component to calculate the putative breakpoints. Since no edges connect split read vertices to the paired read vertices, this analysis can report a single variant as two separate variants, one supported only by split reads, and the other supported only by paired-end reads. The next step in indelMINER involves combining the split read and paired-end read evidence for the same variant. This can be easily accomplished by sorting the variants and then combining the adjacent variants if merging them does not violate the outer distance constraint for the pairs that support the variant.

\section{Filtering and annotation}

The resulting variants can be filtered based on the number of pairs and split-reads that support the variant, the average depth of coverage across the breakpoints, the RMS value of the mapping quality across the breakpoints and the number of reads across the breakpoint that align with a zero mapping quality. We also report the length of the flanking sequences on both sides of the variant for every read that supports the variant. Besides identification, indelMINER can also be used to tag indels with their presence or absence in another sample. This is extremely useful in analysis of normal/tumor pairs and in cases where multiple members of the same family are being analyzed for de-novo variants. This is accomplished by using a list of putative indels in one of the samples "A" (tumor in case of normal/tumor pairs) as input to an instance of indelMINER. indelMINER stores these intervals from sample A, and then processes all improper pairs and split reads in the second sample "B"; including reads with zero mapping qualities, secondary and suboptimal alignments, to find any evidence that can support the breakpoints in "A". This information is used to annotate the variants as being present or absent in sample B.

\section{Additional files}

Additional file 1: The suppmentary methods and figures.

Additional file 2: List of indels chosen for validation and its details.

\section{Competing interests}

The authors declare that they have no competing interests.

\section{Authors' contributions}

AR, WM designed the study and developed indeIMINER. The validations were performed by TLO. AR, WM, TPL wrote the manuscript. All authors read and approved the final manuscript.

\section{Acknowledgements}

We thank Dan Notterman and Lisa Schneper for discussions and suggestions during the development of indeIMINER.

\section{Funding}

This work was supported by funding from Penn State CTSI NIH Grant Number UL1TR000127, and Gordon and Betty Moore Foundation to AR.

\section{Author details}

${ }^{1}$ Center for Comparative Genomics and Bioinformatics, Pennsylvania State University, 506, Wartik Laboratory, University Park, PA 16802, USA. ${ }^{2}$ Department of Medicine, Hematology and Oncology, and the University of Virginia Cancer Center, University of Virginia, Charlottesville, VA 22908, USA. ${ }^{3}$ Department of Public Health Sciences and Center for Public Health Genomics, University of Virginia, Charlottesville, VA 22908, USA.

Received: 18 June 2014 Accepted: 29 January 2015

Published online: 13 February 2015

\section{References}

1. Lam HYK, Pan C, Clark MJ, Lacroute P, Chen R, Haraksingh R, et al. Detecting and annotating genetic variations using the HugeSeq pipeline. Nat Biotechnol. 2012;30:226-9.

2. Stenson PD, Ball EV, Mort M, Phillips AD, Shaw K, Cooper DN. The Human Gene Mutation Database (HGMD) and its exploitation in the fields of personalized genomics and molecular evolution. Curr Protoc Bioinform. 2012;39:1. 13:1.13.1-1.13.20.

3. Li S, Li R, Li H, Lu J, Li Y, Bolund L, et al. SOAPindel: efficient identification of indels from short paired reads. Genome Res. 2013;23:195-200.

4. Ye K, Schulz MH, Long Q, Apweiler R, Ning Z. Pindel: a pattern growth approach to detect break points of large deletions and medium sized insertions from paired-end short reads. Bioinformatics. 2009;25:2865-71.

5. Jiang Y, Wang Y, Brudno M. PRISM: Pair read informed split read mapping for base-pair level detection of insertion, deletion and structural variants. Bioinformatics. 2012;28(20):2576-83.

6. Abyzov A, Urban AE, Snyder M, Gerstein M. CNVnator: An approach to discover, genotype and characterize typical and atypical CNVs from family and population genome sequencing. Genome Res. 2011;21:974-84.

7. Levy S, Sutton G, Ng PC, Feuk L, Halpern AL, Walenz BP, et al. The diploid genome sequence of an individual human. PLoS Biol. 2007;5:e254.

8. Hu X, Yuan J, Shi Y, Lu J, Liu B, Li Z, et al. plRS: Profile-based Illumina pair-end reads simulator. Bioinformatics. 2012;28:1533-5.

9. Li H, Durbin R. Fast and accurate short read alignment with Burrows-Wheeler transform. Bioinformatics. 2009;25:1754-60.

10. McKenna A, Hanna M, Banks E, Sivachenko A, Cibulskis K, Kernytsky A, et al. The Genome Analysis Toolkit: a MapReduce framework for analyzing next-generation DNA sequencing data. Genome Res. 2010;20:1297-303.

11. Li H, Handsaker B, Wysoker A, Fennell T, Ruan J, Homer N, et al. The sequence Alignment/Map format and SAMtools. Bioinformatics. 2009;25:2078-9.

12. Kidd JM, Cooper GM, Donahue WF, Hayden HS, Sampas N, Graves T, et al. Mapping and sequencing of structural variation from eight human genomes. Nature. 2008:453:56-64.

13. Albers C, Lunter G, MacArthur DG, McVean G, Ouwehand WH, Durbin R. Dindel: accurate indel calls from short-read data. Genome Res. 2010:961-973

14. Bentley DR, Balasubramanian S, Swerdlow HP, Smith GP, Milton J, Brown $\mathrm{CG}$, et al. Accurate whole human genome sequencing using reversible terminator chemistry. Nature. 2008;456:53-9.

15. McKernan KJ, Peckham HE, Costa GL, McLaughlin SF, Fu Y, Tsung EF, et al. Sequence and structural variation in a human genome uncovered by short-read, massively parallel ligation sequencing using two-base encoding. Genome Res. 2009;19:1527-41.

16. Pruitt KD, Tatusova T, Brown GR, Maglott DR. NCBI Reference Sequences (RefSeq): current status, new features and genome annotation policy. Nucleic Acids Res. 2012:40(Database issue):D130-5.

17. Mills RE, Pittard WS, Mullaney JM, Farooq U, Creasy TH, Mahurkar AA, et al. Natural genetic variation caused by small insertions and deletions in the human genome. Genome Res. 2011;21:830-9.

18. Mullaney JM, Mills RE, Pittard WS, Devine SE. Small insertions and deletions (INDELs) in human genomes. Hum Mol Genet. 2010;19:R131-6.

19. Chen H, Boutros PC. VennDiagram: a package for the generation of highly-customizable Venn and Euler diagrams in R. BMC Bioinformatics. 2011;12:35.

20. Sokol L, Loughran TP. Large granular lymphocyte leukemia. Curr Hematol Malig Rep. 2007;2:278-82.

21. Loughran TP, Kadin ME, Starkebaum G, Abkowitz JL, Clark EA, Disteche C, et al. Leukemia of large granular lymphocytes: association with clonal chromosomal abnormalities and autoimmune neutropenia, thrombocytopenia, and hemolytic anemia. Ann Intern Med. 1985;102:169-75. 
22. Banerji S, Cibulskis K, Rangel-Escareno C, Brown KK, Carter SL, Frederick AM, et al. Sequence analysis of mutations and translocations across breast cancer subtypes. Nature. 2012;486:405-9.

23. Ratan A, Miller W, Guillory J, Stinson J, Seshagiri S, Schuster SC. Comparison of sequencing platforms for single nucleotide variant calls in a human sample. PLoS One. 2013;8:e55089.

24. Chao KM, Pearson WR, Miller W. Aligning two sequences within a specified diagonal band. Comput Appl Biosci. 1992;8:481-7.

Submit your next manuscript to BioMed Central and take full advantage of:

- Convenient online submission

- Thorough peer review

- No space constraints or color figure charges

- Immediate publication on acceptance

- Inclusion in PubMed, CAS, Scopus and Google Scholar

- Research which is freely available for redistribution 